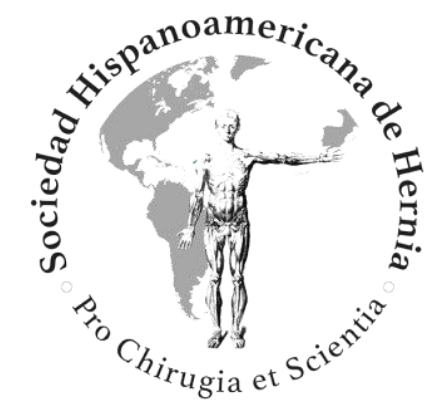

Nueva técnica de hernioplastia para hernia incisional secundaria a catéter de Tenckhoff percutáneo. Resultados preliminares

A novel hernioplasty technique for incisional hernia secondary to percutaneous Tenckhoff catheter. Preliminary results 


\section{OR 370}

\section{Nueva técnica de hernioplastia para hernia incisional secundaria a catéter de Tenckhoff percutáneo. Resultados preliminares}

A novel hernioplasty technique for incisional hernia secondary to percutaneous Tenckhoff catheter. Preliminary results

Luis Manuel García Bravo¹, Gerardo Evaristo Méndez ${ }^{1}$, Héctor Armando Cisneros Muñoz², Juan Carlos Mayagoitia González², Román Indalecio García González ${ }^{1}$, Erika Patricia Guadalupe López Rodríguez ${ }^{1}$

${ }^{1}$ Departamento de Cirugía General. Hospital Regional Dr. Valentín Gómez Farías. Zapopan, Jalisco (México) ${ }^{2}$ Departamento de Cirugía General. Hospital Médica Campestre. León, Guanajuato (México)

Recibido: 03/08/2020

Aceptado: $17 / 08 / 2020$

Autor para correspondencia: Luis Manuel García Bravo. Departamento de Cirugía General. Hospital Regional Dr. Valentín Gómez Farías. Séptimo piso. Av. Soledad Orozco 203, Col. El Capullo. 45100 Zapopan, Jalisco (México) Correo electrónico: drbisonte@hotmail.com

DOI: $10.20960 /$ rhh.00370

Conflicto de interés: los autores declaran no tener conflicto de intereses.

\section{RESUMEN}

Introducción: La diálisis peritoneal es una alternativa de reemplazo para la enfermedad renal crónica. La colocación percutánea suele ser la técnica de 
elección por los nefrólogos y no está exenta de presentar hernias pericatéter que requieran manejo quirúrgico. El objetivo de este trabajo es presentar una técnica quirúrgica alternativa para la reparación de las hernias pericatéter por colocación percutánea.

Material y métodos: Estudio prospectivo, observacional y descriptivo de una serie de casos con pacientes que desarrollaron hernias pericatéter posterior a la colocación percutánea del mismo. Las variables analizadas fueron: sexo, edad, índice de masa corporal (IMC), hipertensión, diabetes, etiología del fallo renal, reparaciones de hernias previas, riesgo anestésico (ASA), complicaciones posoperatorias de la herida quirúrgica o del catéter y recidiva de la hernia.

Resultados: Se operaron 8 pacientes, 5 hombres (63\%) y 3 mujeres (37\%), con hernias pericatéter intervenidos quirúrgicamente con nuestra técnica. El IMC promedio en hombres fue $31 \mathrm{~kg} / \mathrm{m}^{2}$ y en mujeres de $30 \mathrm{~kg} / \mathrm{m}^{2}$. El tiempo quirúrgico promedio fue 78 minutos y el de estancia hospitalaria de 51 horas. El tiempo promedio de seguimiento fue de 13.8 meses sin recidiva de la hernia.

Conclusiones: La técnica quirúrgica que presentamos es una alternativa para reparar la hernia pericatéter secundaria tras la inserción percutánea de catéter Tenckhoff que evita el riesgo de generar otra hernia al reubicar el catéter en un sitio diferente de la pared abdominal.

Palabras clave: Hernia incisional, hernioplastia incisional, catéter Tenckhoff percutáneo, diálisis peritoneal.

\section{ABSTRACT}

Introduction: Peritoneal dialysis is an option for chronic kidney disease. Percutaneous placement is usually the technique of choice for nephrologists, but it is not exempt from pericatheter hernias that require surgical management. The objective of this work is to present an alternative surgical technique for the repair of pericatheter hernias by percutaneous placement. 
Material and methods: Prospective, observational and descriptive study of a series of cases with patients who developed pericatheter hernias after percutaneous placement. The variables analyzed were sex, age, BMI, hypertension, diabetes, etiology of renal failure, previous hernia repairs, ASA, post-operative complications of the surgical wound or catheter and recurrence of the hernia.

Results: Eight patients, 5 men (63\%) and 3 women (37\%) with pericatheter hernias operated with our technique. The average BMI in men was $31 \mathrm{~kg} / \mathrm{m}^{2}$ and in women $30 \mathrm{~kg} / \mathrm{m}^{2}$. The average surgical time was 78 minutes and the average hospital stay was 51 hours. The average follow-up time was 13.8 months with no recurrence of the hernia.

Conclusions: The surgical technique we describe is an alternative to repair the pericatheter hernia secondary to percutaneous Tenckhoff catheter insertion, which avoids the risk of generating another hernia by relocating the catheter to another site in the abdominal wall.

Keywords: Incisional hernia, incisional hernioplasty, percutaneous Tenckhoff catheter, peritoneal dialysis.

\section{INTRODUCCIÓN}

La diálisis peritoneal continua ambulatoria (DPCA) es una alternativa eficaz de reemplazo renal para el tratamiento de la enfermedad renal crónica en etapa terminal ${ }^{1}$. Han sido descritos diferentes procedimientos para la inserción del catéter de diálisis peritoneal (DP), principalmente por abordaje quirúrgico abierto, laparoscópico, peritoneoscópico y percutáneo². En comparación con los otros métodos mencionados, la inserción percutánea puede ofrecer una recuperación y deambulación posoperatoria más temprana, menor coste económico y suele ser la técnica de elección por los nefrólogos cuando no existen contraindicaciones para realizarla ${ }^{3}$. Sin embargo, en términos generales, comparte con los otros abordajes las mismas potenciales complicaciones no infecciosas relacionadas con la 
implantación del catéter, como la formación de hematomas, la fuga del líquido de diálisis, las lesiones viscerales, la disfunción mecánica del flujo de diálisis por migración u obstrucción del catéter y, más raramente, el desarrollo de hernias pericatéter $(\mathrm{HPC})^{4}$. Actualmente, con el uso de una incisión paramediana para la inserción del catéter, la incidencia de las HPC es en promedio de $2.7 \%$ (rango: 0-11 \%) y para incisiones medias con promedio de $10 \%$ (rango: $3.3-17 \%)^{5}$. Las HPC pueden provocar que la DP sea poco eficaz debido a la retención del líquido en el saco herniario, fuga del líquido de diálisis alrededor del catéter, deformidad de la pared abdominal, dolor o malestar en el sitio de la inserción del catéter, así como a la posible incarceración y/o estrangulación intestinal que requiera una operación urgente, por lo que se recomienda su reparación quirúrgica electiva al ser diagnosticadas en pacientes tratados con DPCA ${ }^{5}$. Esta reparación suele efectuarse mediante cierre del defecto herniario y recolocación del catéter en otro sitio de la pared abdominal ${ }^{6}$, pero esto puede provocar otra zona musculoaponeurótica débil de la pared abdominal.

El objetivo de nuestro estudio es presentar una técnica quirúrgica alternativa de fácil ejecución, para la reparación de las HPC secundarias a la colocación percutánea de un catéter tipo Tenckhoff mediante la reimplantación en su sitio original.

\section{MATERIAL Y MÉTODOS}

Estudio prospectivo, observacional y descriptivo entre el 19 de enero y el 14 de noviembre del año 2019, en el departamento de Cirugía General del Hospital Dr. Valentín Gómez Farías, centro hospitalario público con servicios asistenciales de tercer nivel y de enseñanza, de una serie de casos con pacientes consecutivos que desarrollaron HPC posterior a la inserción percutánea de un catéter de diálisis peritoneal. Esta colocación de los catéteres percutáneos para DP se realiza en forma sistematizada por nefrólogos del hospital, con anestesia local, sin guía fluorosocópica o de ultrasonido y modificando de la técnica de Seldinger por una incisión 
paramediana izquierda. Los pacientes fueron reclutados de manera consecutiva en el Servicio de Cirugía General del Hospital. Se incluyeron hombres y mujeres con edad $\geq 18$ años que presentaron una hernia incisional secundaria a la instalación percutánea de un catéter recto de Tenckhoff para DPCA, operados en forma electiva por nuestro grupo quirúrgico.

Fueron excluidos los pacientes menores de 18 años, aquellos cuyo catéter fue colocado por un abordaje diferente al percutáneo, aquellos sometidos a cirugía de urgencia por incarceración o estrangulación intestinal de su hernia, pacientes con infección abdominal o en cualquier otro sitio y los pacientes operados con cualquier otra técnica de reparación que la propuesta en el presente protocolo.

Se realizó en todos los pacientes una tomografía axial computarizada simple de abdomen para descartar otras posibles hernias concurrentes en la pared abdominal, poner de manifiesto la relación entre el catéter con la hernia y determinar sus dimensiones.

Las variables que se analizaron en este grupo fueron: sexo, edad, índice de masa corporal en $\mathrm{kg} / \mathrm{m}^{2}$, comorbilidades como hipertensión arterial, diabetes mellitus, tabaquismo, etiología del fallo renal, reparaciones de hernias previas, clasificación del estado físico ASA (American Society of Anesthesiologists), tiempo quirúrgico en minutos, complicaciones posoperatorias de la herida quirúrgica en los primeros 30 días posoperatorios (seromas, hematomas, infección del sitio quirúrgico) o del catéter (peritonitis, disfunción con o sin fuga del líquido de DP), tiempo de estancia hospitalaria en horas y recidiva de la hernia (clínica o ecográfica).

\section{Descripción de la técnica}

La técnica de reparación de las HPC descrita a continuación, se utilizó en todos los pacientes bajo bloqueo anestésico peridural y administración de antibióticos por vía intravenosa una hora antes del procedimiento quirúrgico:

1. Incisión de la piel sobre la protuberancia de la hernia 
2. Disección y aislamiento del saco herniario hasta su base e incisión circunferencial de la aponeurosis anterior del músculo recto del abdomen para crear un espacio subaponeurótico circunferencial de 3 $\mathrm{cm}$ (fig. 2).

3. Apertura y resección del saco herniario con el catéter de Tenckhoff en su interior, hasta las fibras del músculo recto abdominal (fig. 3).

4. Recambio del catéter de Tenckhoff y verificación de su funcionalidad mediante un recambio con $1000 \mathrm{ml}$ de líquido de DP.

5. Cierre de las fibras del músculo recto abdominal y del peritoneo fijando el cojinete interno de dacrón conjuntamente, con puntos separados de poliglactina 2-0. El catéter sale de la cavidad abdominal en ángulo de $45^{\circ}$ (fig. 4).

6. Recorte de $4 \times 4 \mathrm{~cm}$ de malla de polipropileno pesado con un orificio central para el paso del catéter (fig. 5).

7. Cierre de la apertura longitudinal de la malla que formó el orificio central con un punto simple de polipropileno 2-0 (fig. 6).

8. Colocación de la malla en el espacio subaponeurótico anterior al músculo recto (prerrectal) fijando con 4 puntos transfasciales con sutura de polipropileno 2-0 (fig. 7).

9. Cierre de la aponeurosis anterior del recto abdominal con sutura continua de polipropileno 2-0 (con cuidado de no dejar apretado el catéter de Tenckhoff) (fig. 8).

10. El catéter de Tenckhoff y el cojinete externo se tunelizan en el tejido graso subcutáneo. Cierre del resto de la herida de forma convencional.

En el posoperatorio inmediato se suspendió la DP por 48 horas y se reinició con un volumen de $1000 \mathrm{ml}$ los siguientes 7 días a criterio de los nefrólogos. Los antibióticos se continuaron vía oral por 7 días. La analgesia posoperatoria se controló con paracetamol (500 mg vía oral cada 8 horas durante 3 días). Para la descripción estadística los datos se presentaron como números y porcentajes o medias \pm desviación estándar. El análisis se realizó con un 
paquete estadístico para las ciencias sociales (SPSS 19.0. SPSS Inc., Chicago, EE. UU.).

Este trabajo fue aprobado por el departamento de Enseñanza e Investigación y el Comité de Ética del hospital sede y en conformidad con los lineamientos del expediente clínico expresados en la Norma Oficial Mexicana NOM-004SSA3-2012 y a la Ley General de Salud en Materia de Investigación para la Salud de los Estados Unidos Mexicanos. Se obtuvo consentimiento informado de todos los pacientes.

\section{RESULTADOS}

Durante el periodo de reclutamiento de casos de 10 meses reunimos 8 pacientes, 5 hombres (63\%) y 3 mujeres (37\%) con HPC que fueron intervenidos quirúrgicamente con nuestra técnica. La edad promedio de los hombres fue de 67 años (rango 53-83) y de las mujeres 50 años (rango 3462). El IMC promedio en hombres fue $31 \mathrm{~kg} / \mathrm{m}^{2}$ (rango 28-33) y en mujeres 30 $\mathrm{kg} / \mathrm{m}^{2}$ (rango 28-30). Todos los pacientes tuvieron hipertensión arterial, mientras que la diabetes mellitus se presentó en 6 pacientes $(75 \%)$. La causa de la insuficiencia renal crónica en 5 casos (63\%) fue por nefropatía diabética y en 3 (37\%) por glomerulonefritis crónica. Tres pacientes (38\%) tuvieron el antecedente quirúrgico de reparación con malla de una hernia umbilical o inguinal antes de colocar un catéter para DP. Todos los pacientes con HPC refirieron como manifestaciones de su hernia dolor y aumento de volumen durante la introducción del líquido de DP en el sitio del catéter, así como disfunción de este manifestado por falla en la salida del líquido de diálisis. La tomografía computarizada de abdomen evidenció en todos los pacientes la presencia de un solo defecto herniario pericatéter, el cual medía de media $0.9 \mathrm{~mm}$ (rango: 0.8-11 mm) en su diámetro mayor (fig. 1).

El tiempo quirúrgico para la reparación de las HPC de media fue de 78 minutos (rango: 60-90). No se presentaron incidentes transoperatorios. No hubo infección del sitio quirúrgico ni fugas del líquido de DP en ningún paciente durante el periodo de 30 días de observación. Un paciente (13\%) 
presentó un hematoma subcutáneo en las primeras 24 horas del posoperatorio el cual se manejó mediante compresión externa con su resolución en 4 días. El tiempo de estancia hospitalario del grupo fue de 51 horas de media (rango 48-72). El primer caso de nuestra serie tuvo disfunción por obstrucción del catéter debido a su reperitonización a los 14 meses de haberlo colocado, por lo que se realizó un recambio del catéter por el mismo sitio de inserción sin agregar ningún otro procedimiento. La tabla I muestra otras características y detalles de los pacientes estudiados.

El tiempo promedio de seguimiento para el grupo de estudio fue de 13.8 meses (rango 8 a 18 meses) sin haber presentado recidiva de la hernia hasta el corte del seguimiento.

\section{DISCUSIÓN}

La HPC es una complicación no infecciosa poco frecuente en los pacientes con DPCA que conlleva considerable morbilidad (fugas tardías del líquido de DP, disfunción y extrusión del catéter, infección peritoneal y obstrucción intestinal). Estas complicaciones suelen ser consecuencia de un defecto musculoaponeurótico en la pared abdominal producido por la colocación del catéter de diálisis y del aumento de la presión intraabdominal relacionada con el uso de altos volúmenes de líquido de diálisis, aunque también la edad, el IMC, la etiología del fallo renal, la colocación del catéter en la línea media del abdomen y el antecedente de otras hernias se pueden asociar con su aparición $n^{7,8}$. Cuando es factible, la técnica de elección por los nefrólogos en nuestro hospital para colocar el catéter de Tenckhoff es mediante el acceso por punción percutánea en la línea paramediana izquierda. Sin embargo, en el lapso de 10 meses hemos documentado 8 casos de HPC. Este elevado número en un periodo de tiempo relativamente corto es debido, principalmente, a que la técnica percutánea no cumple con el requisito básico de que el cojinete interno de dacrón quede fijo en el área subaponeurótica9. Por tanto, la técnica percutánea provoca que el revestimiento peritoneal se extienda hacia la capa grasa subcutánea y no a 
lo largo de la superficie del catéter para llegar al cojinete profundo y sellar la abertura peritoneal, creando así la posibilidad de fuga del líquido de diálisis o de una $\mathrm{HPC}^{8}$. En todos nuestros pacientes encontramos el cojinete interno dentro del saco herniario, pero en ningún caso hubo fijación del cojinete al peritoneo, lo cual atribuimos a que la presencia del líquido de diálisis dentro de la hernia evitó su adherencia. Con el fin de exponer el cojinete interno y su recolocación por debajo de la aponeurosis del músculo recto, siempre tuvimos la necesidad de abrir y resecar el saco peritoneal, a pesar de la recomendación de algunos autores de que sea reducido íntegro a la cavidad peritoneal $^{8}$. Con este procedimiento, aunado al cierre cuidadoso y hermético del peritoneo alrededor del catéter y a la amplia superposición de la malla subaponeurótica, no hemos documentado ninguna hernia ni fuga temprana (dentro de los primeros 30 días del posoperatorio), pero también es muy probable que el protocolo que utilizamos con bajos volúmenes de líquido de diálisis en los primeros 7 días del posoperatorio inmediato haya contribuido a ello. Si bien estos principios en el manejo de las HPC (uso de malla y posición del catéter en el plano subaponeurótico) no es diferente de lo recomendado por otros autores ${ }^{10,11}$, no reubicamos el remplazo del catéter de Tenckhoff en un cuadrante diferente del abdomen al de su inserción original, principalmente para respetar otras áreas de la pared abdominal que en el futuro sean intervenidas quirúrgicamente (v. gr., trasplante renal), evitar la creación de otra zona de debilidad musculoaponeurótica con el potencial de favorecer una nueva hernia y para no prolongar el tiempo quirúrgico en su reparación. El abordaje laparoscópico puede fácilmente crear un túnel subaponeurótico para colocar el cojinete interno de dacrón del catéter de Tenckhoff dentro del músculo recto, lo cual disminuye el riesgo en la formación de una HPC ${ }^{12}$. Sin embargo, y específicamente en la reparación de la HPC, consideramos que el procedimiento laparoscópico conlleva la desventajas de la anestesia general, la necesidad de equipo especializado para su realización y un mayor tiempo quirúrgico, sin mejores resultados que el abordaje abierto que presentamos, ya que cada puerto de entrada (por lo 
general, de 2 a 4 puertos) crea un sitio débil en la pared abdominal donde una hernia puede ocurrir y la frecuencia de complicaciones postoperatorias (fuga, desplazamiento, disfunción en el flujo del líquido de DP y obstrucción del catéter), no son diferentes a las que se presentan con la cirugía abierta en general ${ }^{13.14}$.

En el trabajo realizado por Gracia $^{15}$, se menciona que los pacientes con diálisis peritoneal y antecedentes de hernias previas tienen mayor riesgo de desarrollar hernias abdominales una vez que se inicia el tratamiento sustitutivo de diálisis peritoneal, y se recomienda reparar estas hernias antes de iniciar la diálisis peritoneal. De los 146 pacientes que incluye en el trabajo, solo 12 (8.3\%) presentaron hernias posteriores al inicio de la diálisis peritoneal sin reportar hernias incisionales o pericatéter y todas las hernias fueron intervenidas con cirugía, colocando una malla sin recurrencias. En nuestros casos, de los 8 pacientes reportados, la mitad tenía el antecedente de hernia ( 3 umbilicales y 1 inguinal bilateral) que se manejaron quirúrgicamente de igual manera con colocación de malla antes de iniciar la diálisis peritoneal. A diferencia del trabajo de Gracia ${ }^{15}$ nosotros sí encontramos hernias pericatéter en el $100 \%$ de los casos, aunque en el trabajo de Gracia no se especifica si los catéteres de diálisis fueron colocados de forma percutánea o abierta.

Una de las mayores series publicada por Balda ${ }^{16}$ describe, mediante un estudio de cohorte y retrospectivo, el tratamiento y el impacto clínico de las hernias en los pacientes con diálisis peritoneal; reporta 308 pacientes en diálisis de los cuales a 110 se les colocó el catéter de forma percutánea y el resto por cirugía abierta. Se encuentran 73 hernias en 63 pacientes. La hernia umbilical era la más fecuente en un $40 \%$ de los casos (29 pacientes) y se describen 14 casos de hernias incisionales de los cuales el $79 \%$ se relacionaba con la inserción del catéter de diálisis peritoneal. Las hernias en todos los casos fueron reparadas sin tensión y solo registraron 1 complicación posoperatoria (hematoma autolimitado), 4 episodios de peritonitis y 7 hernias recidivaron (10\%), con un tiempo promedio de 
recidiva de 12 meses (rango: 7-32). Lo que no se menciona en este estudio es si en las hernias pericatéter reubicaron el catéter de Tenckhoff en otro sitio distinto al de la hernia. Tampoco mencionan cuántas de las hernias pericatéter reportadas fueron con la técnica percutánea o cirugía abierta para su colocación. Nosotros reportamos un caso de hematoma de igual manera autolimitado, manejado con vendaje compresivo, no presentamos cuadros de peritonitis y en nuestra serie de casos todas las hernias fueron por inserción percutánea del catéter de diálisis. A todos los pacientes se les reparó la hernia pericatéter sin tensión, al mismo tiempo que se recolocó el catéter de Tenckhoff con la técnica descrita en nuestro trabajo. Hasta el corte de nuestro estudio con un seguimiento promedio de 13.8 meses no se han presentado recidivas de la hernia con la técnica descrita.

Dentro de las alternativas quirúrgicas para reparar las hernias en pacientes con diálisis peritoneal existe poca literatura que describa una técnica ideal para reparar hernias en estos pacientes. En lo que coinciden los estudios es en la reparación de estos defectos con técnicas sin tensión. Guzmán ${ }^{17}$, presenta un estudio prospectivo de 50 pacientes en diálisis peritoneal con hernia incisional (media o infraumbilical) proponiendo una técnica quirúrgica donde diseca el saco sin abrirlo, se hacen incisiones longitudinales laterales a los márgenes de la hernia, la cara profunda de la aponeurosis en las incisiones laterales se diseca y se separan del músculo recto y la malla se sutura a la cara profunda con suturas interrumpidas. Se inicia la diálisis peritoneal en el posoperatorio inmediato sin fugas de líquido. Del total de pacientes presentados (50), 4 necesitaron reubicación del catéter de diálisis y 2 retiro por infección, con un periodo de seguimiento medio de 2 años sin recurrencias; a diferencia de la técnica descrita por Guzmán ${ }^{17}$ en nuestro grupo sí resecamos el saco herniario ya que las hernias presentadas en nuestro trabajo son pericatéter y dentro del saco herniario se encuentra el cojinete de dacrón, al ser defectos pequeños (rango: 8-11 mm) nos permiten hacer resección del saco y colocar el catéter de diálisis por el mismo sitio de inserción percutánea. A diferencia de Guzmán que no especifica dónde 
reubica el catéter de diálisis de los pacientes que disfuncionaron, nosotros iniciamos la diálisis peritoneal a las 48 horas del posquirúrgico sin fugas de líquido, de igual manera no hemos tenido recidiva de la hernia aunque el seguimiento de los pacientes es menor.

En otro trabajo presentado por Guzmán ${ }^{18}$ describe dos técnicas de colocación de catéter de diálisis peritoneal (línea media o paramedia) para ver la incidencia de disfunción de catéter y la formación de hernias incisionales; observa que la mayoría de los pacientes presentó complicaciones en la función del catéter durante la primera semana posterior a la colocación; las principales causas fueron la obstrucción por epiplón y fuga de solución dializante sin reportar hernias incisionales en ambas técnicas; el periodo de seguimiento de los pacientes fue de 30 días y observaron mayor disfunción en los catéteres colocados por línea media que los colocados paramedialmente ( $80 \%$ y $37.5 \%$, respectivamente). Se requirió retirar y recolocar los catéteres sin especificar si fue en el mismo sitio o se cambió de lugar. En nuestro trabajo tuvimos la disfunción del catéter en un paciente a los 14 meses de su colocación; el seguimiento por Guzmán es muy corto para ver la aparición de hernias incisionales asociadas al catéter, en comparación con nuestro trabajo que se lleva un seguimiento mayor (rango: 8-18 meses) sin recidivas. Finalmente, nuestros resultados son preliminares por lo que son necesarios estudios adicionales a largo plazo para validar los resultados aquí presentados.

\section{CONCLUSIONES}

La técnica quirúrgica presentada es una alternativa para reparar la HPC secundaria a la inserción percutánea de un catéter de Tenckhoff para pacientes con DPCA. Consideramos que es un procedimiento de bajo riesgo, sencillo de realizar, fiable, seguro y que evita el riesgo de generar otra hernia al reubicar el catéter en otro sitio de la pared abdominal.

\section{BIBLIOGRAFÍA}


1. Tokgoz B. Clinical advantages of peritoneal dialysis. Perit Dial Int. 2009;29 Suppl2:S59-S61.

2. Al-Natour M, Thompson D. Peritoneal Dialysis. Semin Intervent Radiol. 2016;33:3-5.

3. Xie D, Zhou J, Cao X, Zhang Q, Sun Y, Tang L, et al. Percutaneous insertion of peritoneal dialysis catheter is a safe and effective technique irrespective of BMI. BMC Nephrol. 2020;21:199-208.

4. Engeset J, Youngson GG. Ambulatory peritoneal dialysis and hernial complications. Surg Clin North Am. 1984;64:385-92.

5. Yang SF, Liu CJ, Yang WC, Chang CF, Yang CY, Li SY, et al. The risk factors and the impact of hernia development on technique survival in peritoneal dialysis patients: a population-based cohort study. Perit Dial Int. 2015;35:3519.

6. Crabtree JH, Siddiqi RA. Simultaneous Catheter Replacement for Infectious and Mechanical Complications Without Interruption of Peritoneal Dialysis. Perit Dial Int. 2016;36:182-7.

7. Goldstein M, Carrillo M, Ghai S. Continuous ambulatory peritoneal dialysisa guide to imaging appearances and complications. Insights Imaging. 2013;4:85-92.

8. Crabtree JH. Rescue and salvage procedures for mechanical and infectious complications of peritoneal dialysis. Int J Artif Organs. 2006;29:67-84.

9. Winchester JF, Kriger FL. Fluid leaks: prevention and treatment. Perit Dial Int. 1994;14(Suppl.3):S43-8.

10. Martínez-Mier G, Garcia-Almazan E, Reyes-Devesa HE, García-García V, Cano-Gutiérrez $\mathrm{S}$, Mora $\mathrm{R}$, et al. Abdominal wall hernias in end-stage renal disease patients on peritoneal dialysis. Perit Dial Int. 2008;28:391-6.

11. García-Ureña MA, Rodríguez CR, Vega Ruiz V, Carnero Hernández FJ, Fernández-Ruiz E, Vazquez Gallego JM, et al. Prevalence and management of hernias in peritoneal dialysis patients. Perit Dial Int. 2006;26:198-202.

12. Crabtree JH, Fishman A. A laparoscopic method for optimal peritoneal dialysis access. Am Surg. 2005;71:135-43. 
13. Harissis HV, Katsios CS, Koliousi EL, Ikonomou MG, Siamopoulos KC, Fatouros, et al. A new simplified one port laparoscopic technique of peritoneal dialysis catheter placement with intra-abdominal fixation. Am J Surg. 2006;192:125-9.

14. Oliver MJ, Perl J, McQuillan R, Blake PG, Jain AK, McCormick B, et al. Quantifying the risk of insertion-related peritoneal dialysis catheter complications following laparoscopic placement: Results from the North American PD Catheter Registry. Perit Dial Int. 2020;40):185-92.

15. Gracia Toledo M, Borras Sans M, Gabarrell A, Duran J, Fernández Giráldez E. Risk factors for abdominal hernias in patients undergoing peritoneal dialysis. Nefrologia. 2011;31(2):218-9.

16. Balda Sagrario, Power Albert, Papalois Vassilios, Brown Edwina. Impact of hernias on peritoneal dialysis technique survival and residual renal function. Perit Dial Int. 2013;33(6):629-34.

17. Guzmán VG, Zaga I. Abdominal wall hernia repair in patients with chronic renal failure and a dialysis catheter. Hernia. 2001;5:9-11.

18. Guzmán VGG, Jaramillo de la Torre E. Abordaje por línea media o paramedia en la colocación de catéter de Tenckhoff en pacientes con diálisis peritoneal continua ambulatoria. Estudio comparativo. Cir Ciruj. 2004;72:193201.

Tabla I. Características de los pacientes estudiados

\begin{tabular}{|l|l|l|l|l|l|l|l|l|l|}
\hline $\begin{array}{l}\text { Pacie } \\
\text { nte }\end{array}$ & Sexo & $\begin{array}{l}\text { Ed } \\
\text { ad }\end{array}$ & $\begin{array}{l}\text { IM } \\
\text { C }\end{array}$ & $\begin{array}{l}\text { Taba } \\
\text { co }\end{array}$ & $\begin{array}{l}\text { AS } \\
\text { A }\end{array}$ & $\begin{array}{l}\text { Herni } \\
\text { as } \\
\text { previa } \\
\text { s }\end{array}$ & $\begin{array}{l}\text { Tiem } \\
\text { po } \\
\text { cirug } \\
\text { ía } \\
\text { (min) }\end{array}$ & $\begin{array}{l}\text { TEH } \\
\text { (hor } \\
\text { as) }\end{array}$ & $\begin{array}{l}\text { Seguimi } \\
\text { ento } \\
\text { (meses) }\end{array}$ \\
\hline 1 & $\begin{array}{l}\text { Homb } \\
\text { re }\end{array}$ & 83 & 28 & Sí & II & - & 80 & 48 & 17 \\
\hline 2 & $\begin{array}{l}\text { Homb } \\
\text { re }\end{array}$ & 53 & 32 & Sí & II & $\begin{array}{l}\text { Umbilic } \\
\text { al }\end{array}$ & 90 & 72 & 16 \\
\hline 3 & Homb & 70 & 33 & No & II & - & 60 & 48 & 16 \\
\hline
\end{tabular}




\begin{tabular}{|l|l|l|l|l|l|l|l|l|l|}
\hline & re & & & & & & & & \\
\hline 4 & Mujer & 53 & 30 & Sí & II & $\begin{array}{l}\text { Umbilic } \\
\text { al }\end{array}$ & 75 & 48 & 15 \\
\hline 5 & $\begin{array}{l}\text { Homb } \\
\text { re }\end{array}$ & 67 & 32 & No & III & $\begin{array}{l}\text { Inguina } \\
\text { I }\end{array}$ & 90 & 48 & 15 \\
\hline 6 & Mujer & 62 & 30 & No & II & - & 70 & 48 & 11 \\
\hline 7 & $\begin{array}{l}\text { Homb } \\
\text { re }\end{array}$ & 61 & 30 & No & II & - & 85 & 48 & 11 \\
\hline 8 & Mujer & 34 & 30 & No & II & $\begin{array}{l}\text { Umbilic } \\
\text { al }\end{array}$ & 70 & 48 & 9 \\
\hline
\end{tabular}

IMC: índice de masa corporal en $\mathrm{kg} / \mathrm{m}^{2}$; ASA: clasificación de la American Society of Anesthesiologists; TEH: tiempo de estancia hospitalaria.

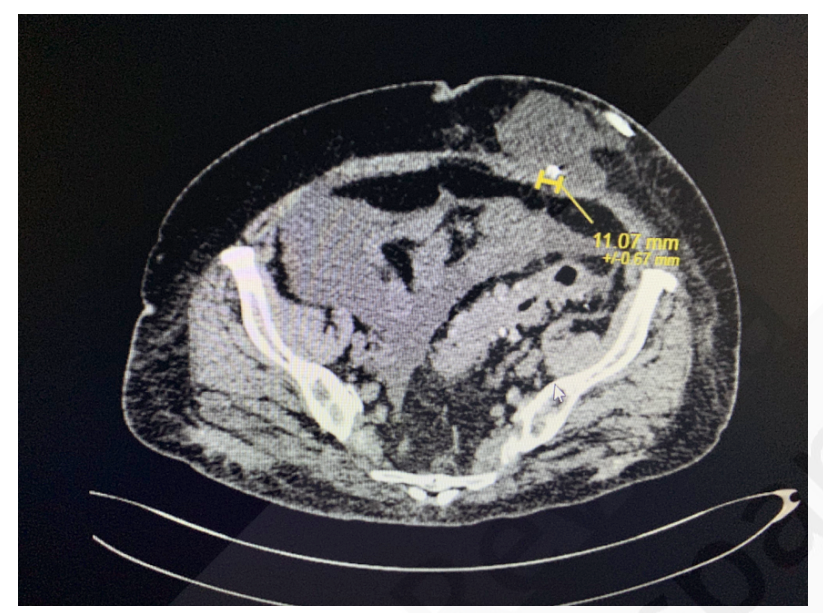

Fig. 1. Hernia pericatéter a través del músculo recto izquierdo.

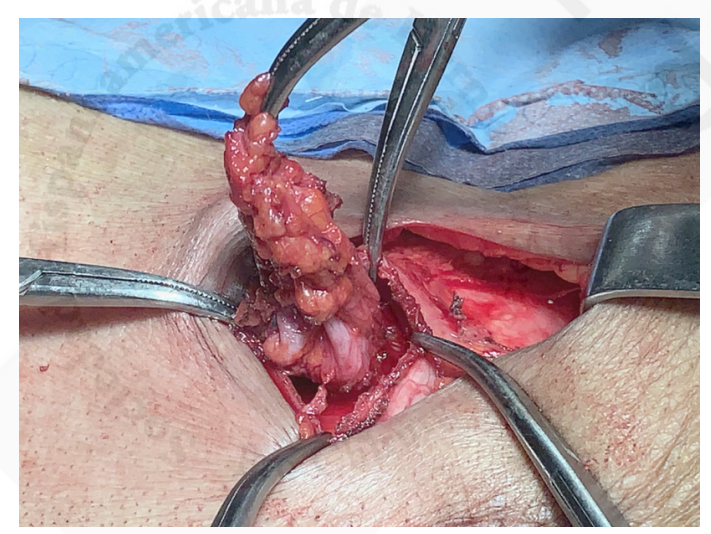

Fig. 2. Disección y aislamiento del saco herniario hasta su base con incisión circunferencial para desarrollar un plano subaponeurótico prerrectal de $3 \mathrm{~cm}$. 


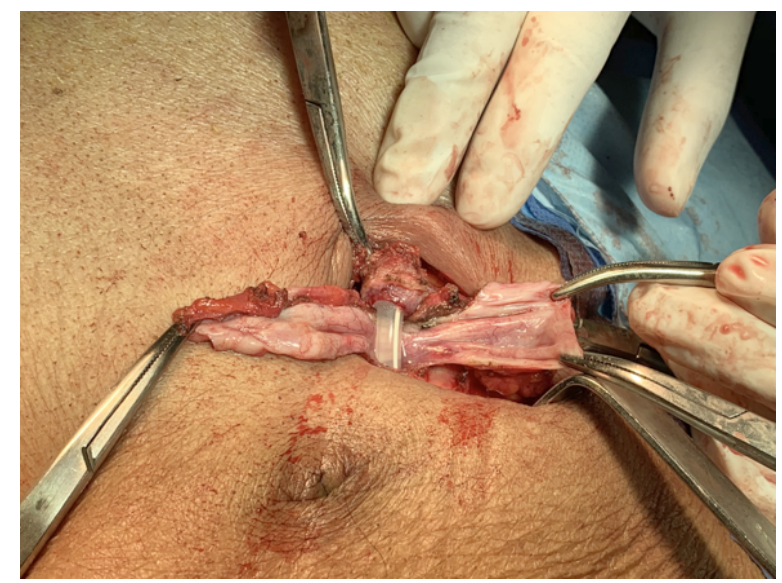

Fig. 3. Apertura y resección del saco herniario con el catéter de Tenckhoff en su interior.

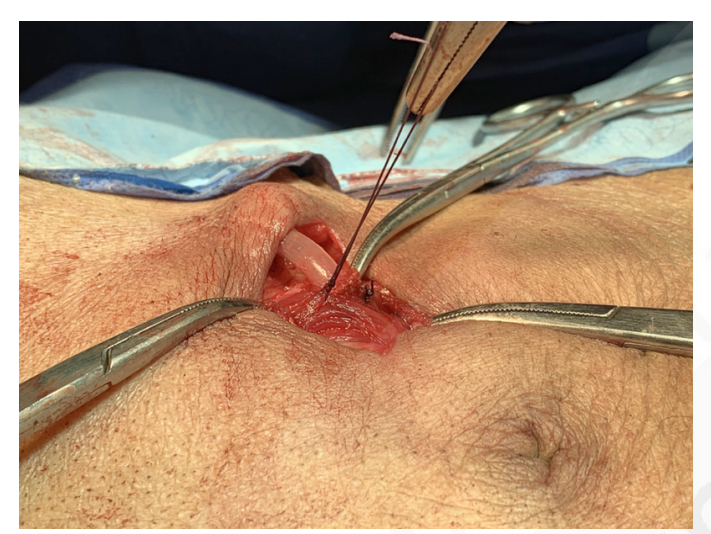

Fig. 4. Cierre de las fibras del músculo recto abdominal y del peritoneo fijando el cojinete interno de dacrón conjuntamente mediante puntos separados de poliglactina 2-0. El catéter sale de cavidad abdominal en ángulo de $45^{\circ}$. 


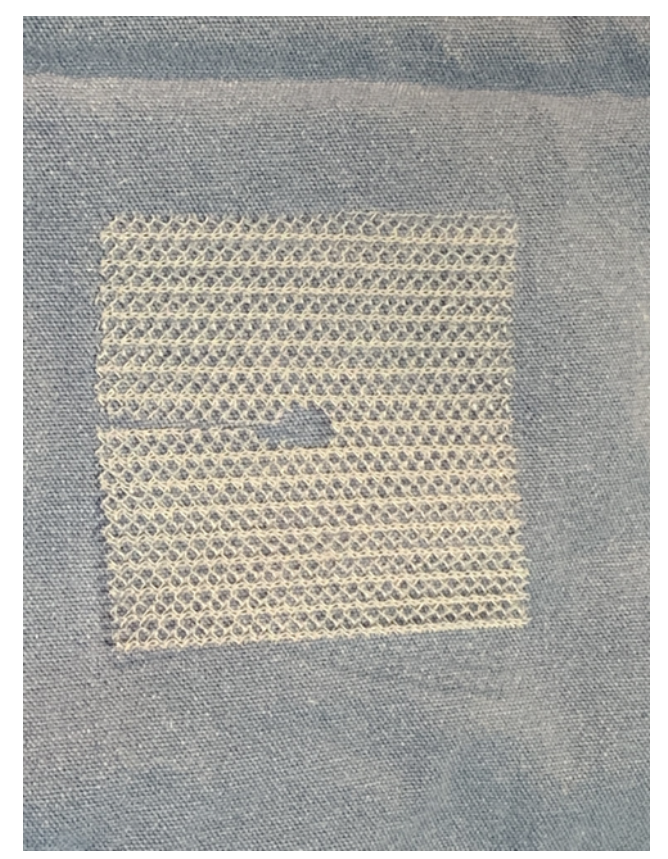

Fig. 5. Malla de polipropileno de aproximadamente $4 \times 4 \mathrm{~cm}$ con orificio central para el paso del catéter.

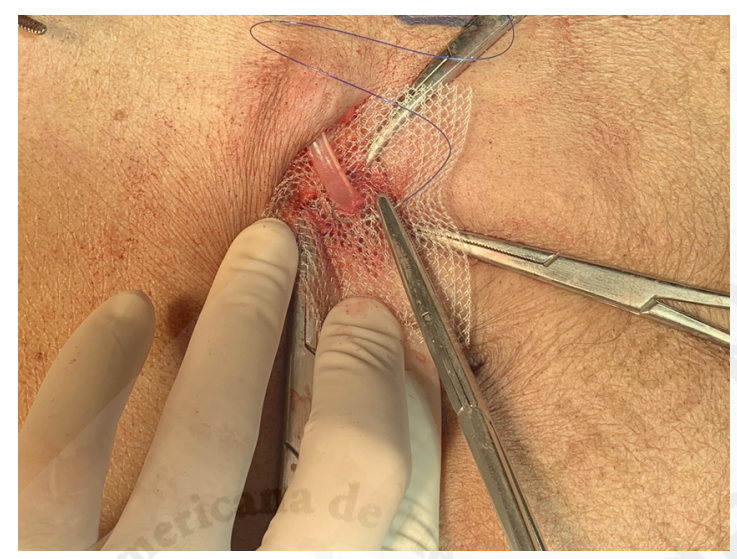

Fig. 6. Cierre del corte longitudinal de la malla con punto simple de polipropileno 2-0. 


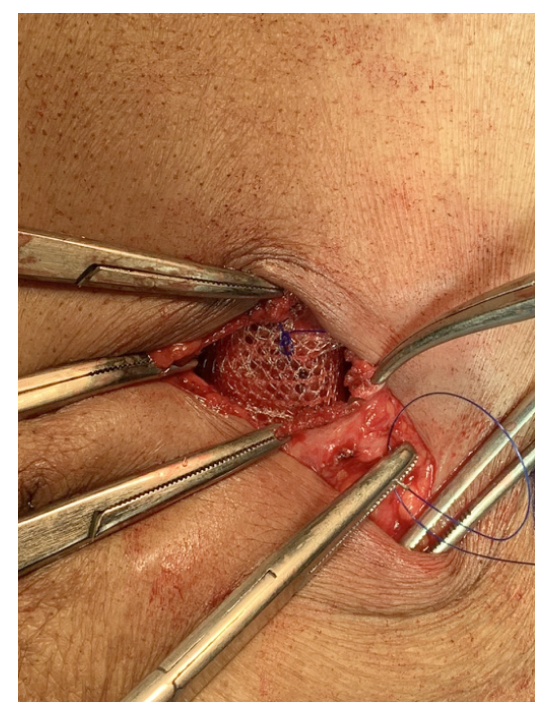

Fig. 7. Colocación y fijación de la malla de polipropileno en el espacio subaponeurótico (prerrectal) con cuatro puntos transfasciales.

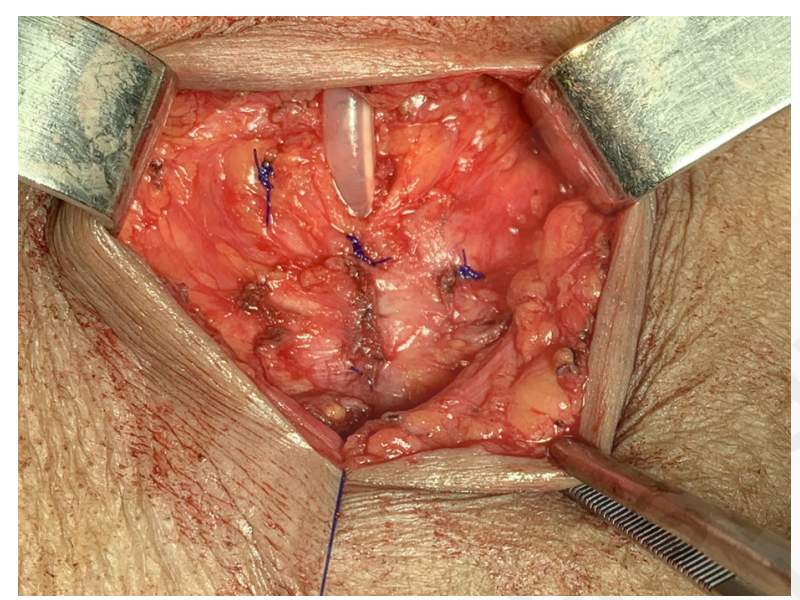

Fig. 8. Cierre de la aponeurosis anterior con sutura contínua de polipropileno 2-0 con catéter Tenckhoff en la parte medial. 